\title{
Cultural differences in neural function associated with object processing
}

\author{
ANGELA H. GUTCHESS, ROBERT C. WELSH, and AYSECAN BODUROǦLU \\ University of Michigan, Ann Arbor, Michigan \\ and \\ DENISE C. PARK \\ University of Illinois at Urbana-Champaign, Urbana, Illinois
}

\begin{abstract}
Behavioral research suggests that Westerners focus more on objects, whereas East Asians attend more to relationships and contexts. We evaluated the neural basis for these cultural differences in an event-related fMRI study. East Asian and American participants incidentally encoded pictures of (1) a target object alone, (2) a background scene with no discernable target object, and (3) a distinct target object against a meaningful background. Americans, relative to East Asians, activated more regions implicated in object processing, including bilateral middle temporal gyrus, left superior parietal/angular gyrus, and right superior temporal/supramarginal gyrus. In contrast to the cultural differences in object-processing areas, few differences emerged in background-processing regions. These results suggest that cultural experiences subtly direct neural activity, particularly for focal objects, at an early stage of scene encoding.
\end{abstract}

Life experiences impact the organization and function of the brain. Structural changes occur in the posterior regions of the hippocampus on the basis of length of time as a London taxicab driver (Maguire et al., 2000), and after merely 3 months of training in juggling, differences were reported in the vicinity of V5 bilaterally and the left posterior intraparietal sulcus (Draganski et al., 2004). Even everyday experience with letters and numbers shapes functional neural organization (Polk \& Farah, 1995; Polk et al., 2002; Puce, Allison, Asgari, Gore, \& McCarthy, 1996). In the present study, we considered the role played by another type of experience - cultural experience-in affecting engagement of neural structures.

Recent behavioral work suggests that culture-specific experiences shape cognition in precise ways. Western cultures place more value on independence and individuality than do Eastern cultures, resulting in an attentional bias toward individual objects, with less regard for context and relationships among items. In contrast, East Asian cultures emphasize interdependent relationships and monitoring of context, resulting in an attentional bias toward

This research was supported by the National Institute on Aging Behavioral Research Program Grant R01 AG015047, the National Institute on Aging Neuroscience and Neuropsychology Program Grant R01AGO6265-15, and an APA Dissertation Research Award. We thank Julie Hautamaki, Hiu-Ying Chen, Heather Huang, Angela Yeung, and the University of Michigan Functional Imaging Center for experimental assistance, and Dick Nisbett, Thad Polk, and Carolyn Yoon for feedback on previous versions of the manuscript. Correspondence concerning this article should be addressed to A. H. Gutchess, Harvard University, William James Hall 868, 33 Kirkland Street, Cambridge, MA 02138 (e-mail: agutchess@wjh.harvard.edu). contextual, relational processing of information (Nisbett, 2003; Nisbett \& Masuda, 2003; Nisbett, Peng, Choi, \& Norenzayan, 2001). These information-processing biases manifest themselves in cognitive behaviors. On a change blindness task, East Asians detect more changes in background contexts, whereas Americans detect more changes in foreground objects (as has been discussed in Nisbett \& Masuda, 2003). On an auditory emotional Stroop task, East Asians experience more interference when required to ignore a conflicting context to make target judgments, whereas Americans experience more interference when basing judgments on context while ignoring the target (Ishii, Reyes, \& Kitayama, 2001). Westerners perform better than East Asians at copying the absolute length of a line, regardless of frame size, whereas East Asians are more accurate than Westerners at reproducing the size of a line relative to its frame (Kitayama, Duffy, Kawamura, \& Larsen, 2003). In addition, Americans recognize previously seen objects in changed contexts better than do Asians, due to their increased focus on object information without regard to context (Chua, Boland, \& Nisbett, 2005; Masuda \& Nisbett, 2001).

The behavioral differences in attentional biases and judgments between Eastern and Western cultures suggest that there should be systematic cultural differences in neural responses to complex scenes. In the present eventrelated functional magnetic resonance imaging (fMRI) study, East Asians and Westerners encoded pictures that differed in the amount of object and context information. When complex scenes that contained both objects and context were studied, we hypothesized that Americans would engage more object-based neural structures, whereas East Asians would engage those specialized for 
contextual processing. Candidate regions for increased object-based processing in Americans included occipital and temporal regions in the ventral "what" stream, as well as the ventral prefrontal cortex (Ungerleider, 1995). The lateral middle temporal cortex, in particular, stores knowledge about object properties and is selectively engaged in the retrieval of semantic knowledge during object perception (Cabeza \& Nyberg, 2000; Devlin et al., 2002; Martin, Wiggs, Ungerleider, \& Haxby, 1996). In contrast to Westerners, Asians might be expected to show increased binding of objects and contexts via the hippocampus or increased encoding of background contexts in the parahippocampal gyrus (Bar, 2004; Goh et al., 2004).

\section{METHOD}

\section{Participants}

Eleven Americans ( 5 of them male) and 11 East Asians ( 6 of them male) participated in the study. Right-handed participants between the ages of 18 and 29 years were recruited and screened for contraindications for scanning, use of drugs known to affect blood flow or brain function, and psychiatric or neuropsychological abnormalities. In addition, in order to qualify for the East Asian group, the East Asian participants must have spent less than 5 years in the United States (average $=2.5$ years, $S D=1.6$ ) and be fluent in Mandarin. Five of the East Asian participants were from Hong Kong, and 6 were from mainland China. The American participants were American born, had spent most of their lives in the United States (22.3 years, $S D=3.0$ ), were native English speakers, and were not of Asian descent. Each participant completed two experimental sessions (pretest and actual scan) and received \$75. The University of Michigan Medical School Institutional Review Board approved the study. All the participants provided written consent.

\section{Behavioral Methods}

In a pretest session, the participants completed a demographic questionnaire and two speed-of-processing tasks, presented in English or Mandarin: dot matching, based on the WAIS-R digit-symbol task (Wechsler, 1981), and pattern matching (Salthouse, 1996). In addition, the participants completed English (WAIS-R; Wechsler, 1981) or Mandarin (WAIS-RC; Gong, 1983) instruments assessing general world knowledge that were specifically normed with culture-appropriate questions. Past research (e.g., Hedden et al., 2002; Park et al., 2005) on types of neuropsychological tests appropriate for cross-cultural comparisons governed the battery creation. East Asian and American participants were matched on age and years of education and performed equivalently on speed-ofprocessing (dot and pattern matching) and WAIS-Information tasks. Therefore, cultural differences could not be attributed to differences
Table 1

Participant Characteristics

\begin{tabular}{lcc}
\hline \multicolumn{1}{c}{ Characteristic } & Americans & Asians \\
\hline Age & 23.27 & 23.00 \\
Years of education & 15.55 & 15.23 \\
Pattern matching & 19.63 & 19.77 \\
Dot matching & 18.18 & 17.00 \\
WAIS Info (scaled) & 12.45 & 11.27 \\
WAIS Comp (scaled) & 10.27 & 12.00 \\
\hline
\end{tabular}

*Signigicant main effect of culture at $p<.05$.

in cognitive ability across the samples. Demographics and battery performance are presented in Table 1. WAIS scores are scaled to allow comparison across samples.

Scanning occurred during encoding, in which 120 pictures and 60 fixation cross baselines were presented for $4 \mathrm{sec}$ each. Across participants, three pseudorandom trial orderings were used. Orders were determined on the basis of random draws from a distribution that optimized signal detection, subject to the constraint that there should be a maximum of 7 picture trials and 3 trials from a single condition in a row. Interstimulus intervals ranged from 0 to $12 \mathrm{sec}$ and contained a black fixation cross. These baseline trials occurred in 4-sec intervals to create variable "jittered" block lengths, with a maximum of $12 \mathrm{sec}$ and an average of approximately $8 \mathrm{sec}$. The picture-processing trials were distributed across two runs, each lasting $6 \mathrm{~min}$ and consisting of 90 encoding trials (60 pictures and 30 baselines per run). Figure 1 presents examples of the pictures presented at encoding. Of the 120 pictures, 40 pictures consisted of only a central target object presented against a white background (object only), 40 consisted of a background only with no central target object (background only), and 40 consisted of a target object against an environmentally meaningful background (combined). The pictures consisted of several types of objects, including animals, office or household objects, foods, and modes of transportation, and were obtained from commercially available photo CDs or online photo databases or were created using a digital camera. The selection of common, everyday objects and scenes was emphasized; pictures were not highly arousing or emotionally valenced and did not include human faces. The pictures were modified by adding or removing objects and backgrounds to create three different versions of each picture. The stimuli were counterbalanced so that each picture was encoded in all three conditions (object only, background only, and combined) across participants. The task was presented using E-Prime software (Psychology Software Tools Inc., Pittsburgh) and the IFIS 9.0 system (MRI Devices Corp., Waukesha, WI). During the 4-sec presentation interval, the participants rated how pleasant they found the picture, using a keypress that corresponded to a rating of pleasant, neutral, or unpleasant. The ratings task was selected to support deep encoding without biasing the participants' attention to the object or the background. Many commonly used deep-encoding

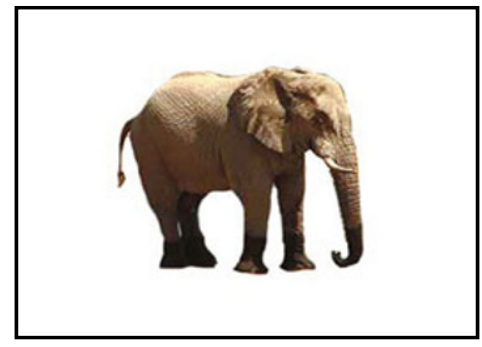

Object-Only Picture

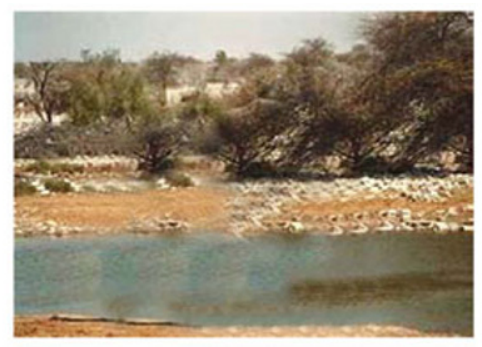

Background-Only

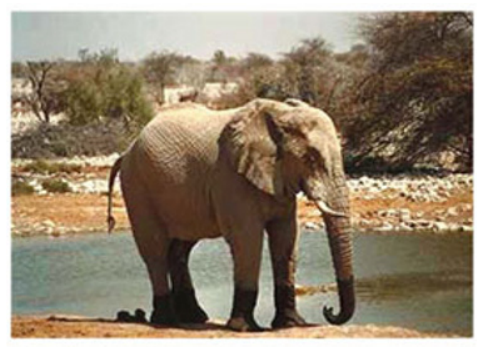

Combined Picture

Figure 1. Example stimuli. 
tasks (such as living/nonliving or common/uncommon) appeared likely to encourage judgments about the object, rather than about the background. Pleasantness ratings across the picture types did not interact with culture. After a 10-min delay, the participants completed a surprise recognition test on the 120 pictures presented earlier in the study, as well as on 120 new distractor pictures (40 targets and 40 lures were tested from each of the three classes of pictures). For each picture, the participants decided whether they had seen the picture in the first part of the study and responded with a yes or no keypress.

\section{Imaging Methods}

Volunteers performed the cognitive task in a full-body 3T GE scanner (General Electric, Milwaukee, WI) outfitted with a standard headcoil, while functional data were acquired with a gradient-echo spiral sequence $(3.0 \mathrm{~T}$ GE LX, TR $=2,000 \mathrm{msec}$, TE $=25 \mathrm{msec}$, $\mathrm{FA}=80, \mathrm{FOV}=20 \mathrm{~cm})$. Thirty-two functional slices, encompassing the entire cortex and cerebellum, were acquired approximately parallel to the anterior-commisure-posterior-commisure line. Each was $4 \mathrm{~mm}$ thick (skip $=0)$, with an effective matrix of $64^{2}(3.125 \times$ $3.125 \mathrm{~mm}$ ). In addition, a high-resolution 3D-SPGR volume was acquired $(0.9375 \mathrm{~mm}$ in-plane resolution, 1.5 -mm-thick slice) for use in normalization to a standard template, as well as structural T1 images that were coplanar with the $\mathrm{T} 2 *$ images.

Using an 8-point Hanning windowed sinc interpolation implemented in $\mathrm{C}++$, fMRI data were slice time corrected. AIR 3.08 was used to perform intrasubject motion correction (Woods, Cherry, \& Mazziotta, 1992). Images were resampled to $3-\mathrm{mm}^{3}$ voxels, converted into the MNI system for standard anatomical localization, and smoothed with a 6-mm Gaussian kernel, using SPM 99 (Wellcome Department of Cognitive Neurology, London). Once the combined picture minus baseline contrasts had been estimated, they were further smoothed with an 8-mm Gaussian kernel, resulting in total effective smoothing of $10 \mathrm{~mm}$.

Individual analysis implemented a model of four regressors in SPM 99: background-only items, object-only items, combined items, and baseline. Contrast images of interest were calculated as the difference in the beta images. To investigate the hypothesis that cultures showed differences in engagement of neural structures for objects and background, we first created two masks: one object regions mask containing regions more active for objects than for backgrounds (object only minus background only) and one background regions mask (background only minus object only). The masks were defined as the union of object regions for the object mask and as the union of background regions for the background mask for either East Asians or Americans and contained voxels significantly active at an uncorrected $p<.001$. Given the novelty of cross-cultural investigations, the ability to label regions as related to relatively greater object or background processing aided interpretation of potential cultural differences (i.e., whether differences arise from object or background processing). We then conducted two-sample random effects group analyses (Holmes \& Friston, 1998) to test for cultural differences (Americans vs. East Asians) in the mask of object regions separate from the mask of background regions. To avoid testing for cultural differences in object or background processing using the same conditions (i.e., object only and background only) as those used to define the masks, we focused on the contrast of combined pictures (stimuli containing both object and background) versus baseline (fixation cross). This approach allowed us to isolate any bias toward engaging background-processing areas or objectprocessing areas when the combined pictures were studied. The use of orthogonal contrasts to restrict search space offers increased sensitivity in detecting cultural differences. Because of the dearth of literature on neural differences across cultures, this approach offers advantages over defining regions of interest a priori, because the whole brain serves as the initial search space. Within each of the two masks (object regions or background regions), cultural differences for the combined picture minus baseline contrast were thresholded at $p<.005$ (uncorrected for multiple comparisons) and a spatial extent of $>5$ voxels. Typically, whole-brain corrections meet at least a threshold of $p<.001$ (uncorrected). However, we adopted a more lenient threshold, because masking reduced the search space to less than 6,000 voxels $(4,693$ voxels for the object mask and 5,669 voxels for the background mask), in contrast to a whole-brain search space of 140,740 voxels.

Despite having reduced the multiple comparisons problem by greater than twenty-fold through the use of masks, we also calculated cluster extent thresholds (Slotnick, Moo, Segal, \& Hart, 2003). Cluster extents were calculated separately for the object and the background masks, using Monte Carlo simulations in volumes approximating the size of each mask. These simulations suggest that clusters consisting of at least 21 voxels in the object mask and 22 voxels in the background mask correct for multiple comparisons at $p<.05$. For the sake of completeness, clusters containing at least 5 voxels that did not surpass the thresholds are also reported, but we urge caution in the interpretation of these smaller clusters.

\section{RESULTS}

\section{Behavioral Results}

There were no significant differences in recognition performance across cultures, using $A^{\prime}$, which incorporates hit and false alarm rates into a single nonparametric measure (Snodgrass \& Corwin, 1988). In a repeated measures ANOVA with culture as a between-group variable and picture type (combined picture, object only, or background only) as a within-subjects variable, only the main effect of picture type was significant $[F(2,40)=27.75, p<.001]$, with the highest performance occurring on the combined and object-only pictures and lowest performance on the background-only pictures (see Table 2). The equivalent performance on the task as a function of culture precludes differences in behavioral performance as the basis for any differences observed in neural engagement and suggests that cultural differences in neural activations reflect strategy differences, rather than effects of difficulty. ${ }^{1}$

\section{Neuroimaging Results}

Within the object-only mask, the contrast revealed considerable cultural differences. As is shown in Table 3A, the Americans activated more regions within the objectspecific mask than did the East Asians, consistent with our hypothesis. Most of these differences occurred in posterior cortical regions, including areas of the left superior parietal gyrus extending into the angular gyrus, the right superior temporal gyrus extending into the supramarginal gyrus, and the left middle temporal gyrus (see Figure 2). Although they did not surpass the cluster extent threshold, activations in the right middle temporal and right insula, the only frontal activation, were also noted. The only object-processing region more active for the East Asians than for the Americans was a small region of the left pos-

Table 2

\begin{tabular}{lcc}
\multicolumn{3}{c}{$\boldsymbol{A}^{\prime}$ by Culture and Picture Type } \\
\hline \multicolumn{1}{c}{ Picture Type } & Americans & Asians \\
\hline Object only & .96 & .94 \\
Background only & .90 & .88 \\
Complex pictures & .95 & .95 \\
\hline
\end{tabular}


Table 3

Cultural Differences for Object- and Background-Processing Regions

\begin{tabular}{|c|c|c|c|c|c|c|}
\hline \multirow[b]{2}{*}{ Activation Region } & \multirow[b]{2}{*}{ Brodmann Area } & \multicolumn{3}{|c|}{ MNI Coordinates } & \multirow[b]{2}{*}{$t$ Score } & \multirow[b]{2}{*}{ No. of Voxels } \\
\hline & & $x$ & $y$ & $z$ & & \\
\hline \multicolumn{7}{|c|}{ A. Object-Processing Regions } \\
\hline \multicolumn{7}{|l|}{ Americans $>$ East Asians } \\
\hline Left superior parietal/ & 7 & -36 & -69 & 54 & 4.51 & $70^{*}$ \\
\hline left angular gyrus & 39 & -48 & -72 & 45 & 4.05 & \\
\hline Left middle temporal & $21 / 20$ & -51 & -42 & -6 & 4.37 & $45^{*}$ \\
\hline Left middle temporal & 20 & -42 & -24 & -9 & 3.72 & $22^{*}$ \\
\hline Right middle temporal & 21 & 57 & -42 & 6 & 3.32 & 13 \\
\hline & & 48 & -33 & -3 & 3.23 & \\
\hline $\begin{array}{l}\text { Right superior temporal/ } \\
\text { supramarginal gyrus }\end{array}$ & $42 / 48 / 41$ & 54 & -33 & 18 & 4.07 & $79^{*}$ \\
\hline Left putamen/caudate & 25 & -18 & 9 & 0 & 3.86 & $31^{*}$ \\
\hline & & -9 & 15 & 0 & 3.48 & \\
\hline Right caudate/palladium & & 15 & 21 & 0 & 3.63 & $26^{*}$ \\
\hline & & 18 & 9 & 0 & 3.45 & \\
\hline Right insula & 48 & 33 & -18 & 12 & 3.43 & 6 \\
\hline Midline & N/A & 0 & -30 & 15 & 3.42 & 8 \\
\hline \multicolumn{7}{|l|}{ East Asians $>$ Americans } \\
\hline Left posterior midtemporal & $21 / 37$ & -60 & -66 & 3 & 3.78 & 10 \\
\hline \multicolumn{7}{|c|}{ B. Background-Processing Regions } \\
\hline Americans $>$ East Asians & & & & & & \\
\hline Left superior occipital & $18 / 19$ & -18 & -81 & 33 & 4.19 & $36^{*}$ \\
\hline Right superior occipital & 19 & 30 & -87 & 27 & 3.17 & 8 \\
\hline East Asians $>$ Americans & & & & & & \\
\hline Left occipital/fusiform & $37 / 19$ & -33 & -60 & -12 & 3.30 & 8 \\
\hline
\end{tabular}

terior middle temporal cortex, but this did not meet the cluster extent threshold.

Cultural differences within the background-processing mask were less pronounced. The Americans activated a region of the left superior occipital cortex more than did the East Asians (see Figure 3A). The homologous region on the right was also present at a subthreshold level. The East Asians activated a region on the border of the left occipital cortex and fusiform more than did the Americans, but this did not achieve significance at the cluster level (see Figure 3B).

\section{DISCUSSION}

The Americans' increased object-based processing is consistent with the finding that Americans attend to individual objects more than do East Asians (Ishii et al., 2003; Kitayama et al., 2003; Masuda \& Nisbett, 2001; Nisbett et al., 2001; Nisbett \& Masuda, 2003). Areas activated more by Americans when complex scenes are processed include the left middle temporal gyrus (BA 20/21), an area involved in retrieval of semantic knowledge about objects (Cabeza \& Nyberg, 2000; Devlin et al., 2002; Martin et al., 1996). The angular gyrus (BA 39) was also preferentially employed by the Americans, and it is engaged by processing cross-modal information (Gernsbacher \&
Kaschak, 2003), consistent with the ability of pictures to cue access of verbal semantic knowledge. The Americans also activated the right superior temporal/supramarginal and superior parietal gyrus more than did the Asians. These regions respond to spatial information (Cabeza \& Nyberg, 2000; Ungerleider, 1995) in tasks including location judgments (Aguirre \& D'Esposito, 1997) and maintenance of spatial information in working memory (Postle, Awh, Jonides, Smith, \& D'Esposito, 2004). Sommer, Rose, Weiller, and Büchel (2005) found that superior parietal activation tracked the success of the encoding of object locations. The involvement of these regions within the object-processing mask may suggest that Americans are more analytic about object features, attending more to the semantic and spatial properties of the focal object than do East Asians. Although not object-processing regions per se, portions of the basal ganglia and insula (albeit subthreshold) also revealed cultural differences within the object-processing mask, suggesting more emotional processing of the objects on the part of the Americans (Phan, Wager, Taylor, \& Liberzon, 2002). Because these regions were contained in the object masks, objects likely contributed to participants' pleasantness ratings for the combined pictures more than do backgrounds, consistent with the relatively neutral and homogenous nature of the backgrounds (e.g., landscapes or office settings). There- 


\section{Cross-Cultural Differences in Object-Processing Regions}

Americans $>$ East Asians

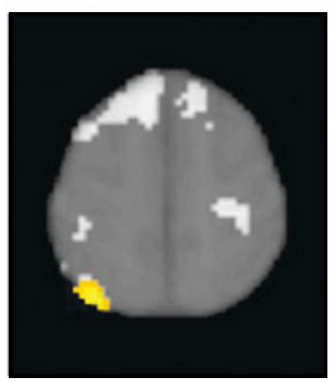

$z=54$

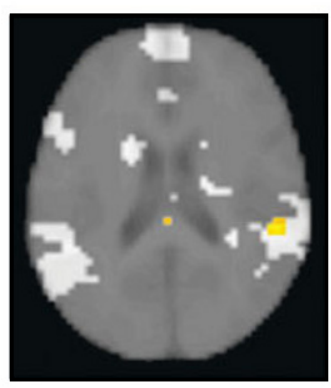

$z=18$

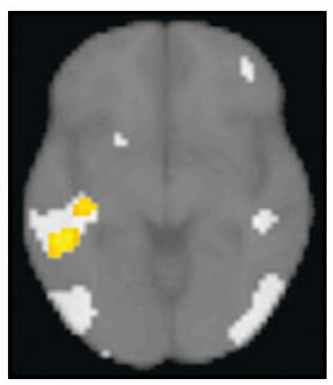

$z=-6$
East Asians > Americans

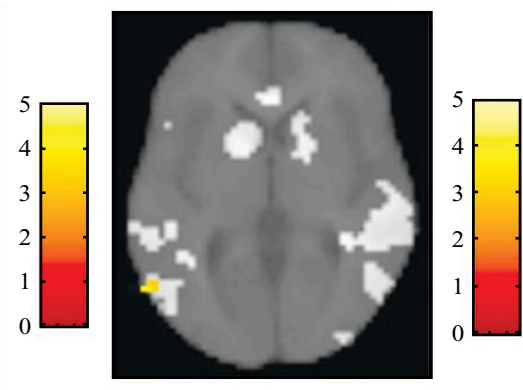

$z=3$

Figure 2. Cultural differences in object-processing regions were tested for the picture minus baseline contrast in the white masked regions. For object-processing regions, the figure shows left superior parietal, right superior temporal/ supramarginal gyrus, and left middle temporal lobe activations that were greater for the Americans than for the East Asians. The rightmost panel displays the greater activation for East Asians in the left midtemporal/fusiform gyrus. The figures display only those voxels significant above the $t$ threshold of 2.85 that occur in clusters of at least 5 voxels.

fore, greater activation of emotion-processing regions by the Americans is in line with their greater focus on objects when judgments about combined pictures are made. In contrast to the widespread regions more involved in object processing for Americans, a single region emerged as more involved for East Asians than for Americans. Although the region is limited in extent and below the cluster extent threshold, it is tempting to draw comparisons with a similar region implicated in the abstraction of semantic knowledge at the categorical level (Grossman et al., 2002). The involvement of this region could reflect East Asians' consideration of an item beyond the specific presented exemplar, perhaps in terms of its relation to other items or extraction of gist, rather than specific perceptual details.

In terms of background-related processing, the East Asians' fusiform involvement is consistent with roles

\section{Cross-Cultural Differences in Background-Processing Regions}

A. Americans $>$ East Asians $(x=-18)$

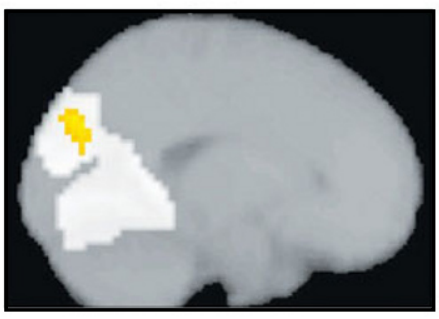

Americans

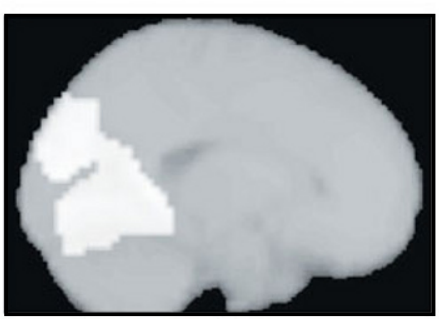

East Asians
B. East Asians $>$ Americans $(x=-33)$

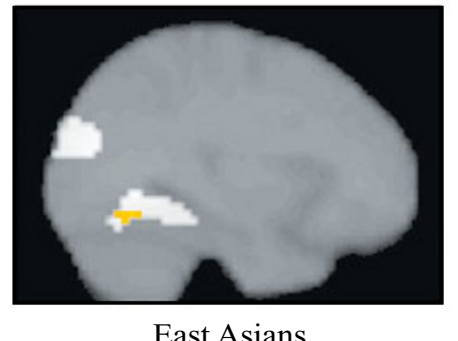

East Asians

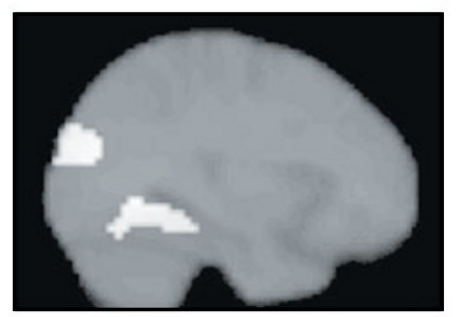

Americans

Figure 3. Cultural differences in background-processing regions were tested for the picture minus baseline contrast in the white masked regions. The left panel (A) illustrates the left superior occipital activation that was greater for the Americans than for the East Asians. The right panel (B) displays the greater activation for the East Asians in the left occipital/fusiform gyrus. 
in perceptual analysis of structure (Joseph \& Gathers, 2003) and viewing of object-background conjunctions (Goh et al., 2004). However, further evidence is needed to substantiate this difference, given the small, nonsignificant cluster size. The BA 19 region activated more by Americans is a perceptual and imagery region, related to analysis of spatial properties (Cabeza \& Nyberg, 2000). Although superior parietal regions also respond to spatial properties, parietal activations likely reflect object-based encoding processes, whereas the occipital region responds to context-driven perceptual analysis. However, inferences about background processing should be made cautiously, given the limited extent of the activations.

In line with our finding of more activity in the middle temporal gyrus for Americans and in the fusiform for East Asians, Whatmough, Chertkow, Murtha, and Hanratty (2002) have proposed a distinction between the roles of the middle temporal gyrus and the fusiform gyrus. They found that both regions were activated by an animal-naming task, but only left middle temporal activations varied as a function of the participants' familiarity with the animals. On the basis of this finding and the response of the fusiform to perceptual processing of "possible" nonsense objects, which by definition do not contain semantic information (Martin et al., 1996), Whatmough et al. (2002) suggested that the fusiform gyrus is involved in the structural processing of complex configurations or distinct perceptual aspects (such as the scene backgrounds in the present study), rather than semantic features of objects. Using this framework, our findings suggest that Westerners engage the middle temporal gyrus more than do East Asians to accomplish semantic processing of complex scenes. In contrast, East Asians may conduct more perceptual processing via the fusiform to encode pictures, without extensive activation of property-specific, semanticprocessing regions.

The results from behavioral studies may be taken to suggest that additional processing of context on the part of East Asians drives cultural differences. However, the neuroimaging results from the present study reveal that cultural differences in the encoding of complex scenes result predominantly from additional processing of objects by Westerners. As has been noted by Park and Gutchess (2002), cultural differences could arise through functional or structural mechanisms. That is, different neural substrates, perhaps reflecting strategy differences, could be involved in accomplishing a task. However, repeated use of a particular circuit could lead to structural reorganization. Functional differences could be further subdivided into those that reflect the use of distinct pathways, as opposed to varying the weighting of the subprocesses along the same pathway. All of these mechanisms could support cultural differences, and further research will be necessary to discern the contributions of each.

The locus of our object-processing activations supports claims that semantic information, particularly about objects, is available after brief presentations of scenes
(Davenport \& Potter, 2004). However, the "special status" afforded objects (Davenport \& Potter, 2004) may reflect the information-processing biases of Westerners, which may not be as evident for East Asians. In this study, we investigated the natural biases of Eastern and Western participants during picture viewing and did not instruct them in a specific encoding strategy. It is possible that directing encoding strategy could minimize cultural differences, although Davenport and Potter have noted the dominance of objects in picture perception across a variety of backgrounds and task instructions.

Our findings suggest that individual differences in experience and strategy at the level of culture can affect neural recruitment relatively soon after perceptual processing. Recent reports of cross-cultural differences in attention that occur within short time windows converge with our finding of cultural differences early in the processing stream. Relative to East Asians, Americans make more fixations to objects within the first $300 \mathrm{msec}$ of picture presentation (Chua et al., 2005), whereas Asians attend to a larger spatial region than do Americans during encoding displays presented for a mere $150 \mathrm{msec}$ (Boduroğlu, Shah, $\&$ Nisbett, 2005).

The finding of low-level encoding differences across cultures could also explain the absence of cultural differences in medial temporal regions. Sommer et al. (2005) have highlighted the direct connections between the superior parietal cortex and the parahippocampal gyrus, and this suggests that cultural differences could occur at an earlier stage of object-based processing, rather than a later stage integrating objects with elaborated contexts. The finding of intact object processing in patients with parahippocampal lesions also demonstrates that object encoding is a more fundamental process, occurring before the encoding of complex scene geometry via the parahippocampus (Epstein, DeYoe, Press, Rosen, \& Kanwisher, 2001). Our data suggest that cultural differences occur prior to scene analysis by the parahippocampal gyrus, and at that stage, encoding processes converge across cultures.

It is likely the case that object bias is an individual difference found to varying degrees in both cultures but is more common in Western than in Asian populations. Individual differences, and the underlying cognitive processes and neural mechanisms, are important to understand, because they can account for individuals' ability to learn new information. This is illustrated by Epstein, Higgins, and Thompson-Schill's (2005) finding that navigational ability determines the importance of viewpoint - and that there is a corresponding involvement of the parahippocampal gyrus - in scene perception. Although culture may influence the cognitive lens automatically employed, individuals' processing biases can be shifted. Priming with Western or East Asian primes leads participants to display stronger biases on social and cognitive tasks consistent with the primed culture (Gardner, Gabriel, \& Lee, 1999; Hong, Morris, Chiu, \& Benet-Martinez, 2000; Miyamoto, Nisbett, \& Masuda, 2006). However, we claim that the 
cultural differences reported in this study represent naturally occurring tendencies in scene encoding that vary across cultural groups.

Even though many cultural differences are flexible to some degree, they appear to be acquired early in life (Chiu, 1972; Fernald \& Morikawa, 1993; Imai \& Gentner, 1997) and operate pervasively across a number of testing environments and tasks. Different information-processing biases across cultures could result from environmental affordances present in one's daily environment. Eastern environments contain more objects than do American environments, which may make the distinction between object and background less clear for East Asians (Miyamoto et al., 2006). Boduroğlu et al. (2005) have suggested that East Asians may attend more broadly to their environment, so that their frame of reference may include several objects. On the other hand, the narrower lens for the Americans may correspond more closely to a single target object. This type of focus would explain the special status of objects in cognitive processing (Davenport \& Potter, 2004), as well as our finding of more distinct processing of objects for Americans.

In conclusion, our findings suggest that differences in object processing contribute to cultural differences in picture encoding. These findings are the first to demonstrate that culture affects neural function when nonverbal stimuli are processed, revealing that cultural experiences shape the engagement of neural systems associated with learning and memory.

\section{REFERENCES}

Aguirre, G. K., \& D'Esposito, M. (1997). Environmental knowledge is subserved by separable dorsal/ventral neural area. Journal of Neuroscience, 17, 2512-2518.

BAR, M. (2004). Visual objects in context. Nature Reviews Neuroscience, $5,617-629$

BoduroğLu, A., Shah, P., \& Nisbett, R. E. (2005, May). Cultural differences in visuospatial working memory and attention. Paper presented at the Midwestern Conference on Culture, Language, and Cognition, Evanston, IL.

Cabeza, R., \& NyberG, L. (2000). Imaging cognition: II. An empirical review of 275 PET and fMRI studies. Journal of Cognitive Neuroscience, 12, 1-47.

ChiU, L.-H. (1972). A cross-cultural comparison of cognitive styles in Chinese and American children. International Journal of Psychology, 7, 235-242.

Chua, H. F., Boland, J. E., \& Nisbett, R. E. (2005). Cultural variation in eye movements during scene perception. Proceedings of the National Academy of Sciences, 102, 12629-12633.

DavenPort, J. L., \& PotTer, M. C. (2004). Scene consistency in object and background perception. Psychological Science, 15, 559-564.

Devlin, J. T., Russell, R. P., Davis, M. H., Price, C. J., Moss, H. E., FADILI, M. J., \& TYLER, L. K. (2002). Is there an anatomical basis for category-specificity? Semantic memory studies in PET and fMRI. Neuropsychologia, 40, 54-75.

Draganski, B., Gaser, C., Busch, V., Schuierer, G., Bogdahn, U., \& MAY, A. (2004). Changes in grey matter induced by training. Nature, 427, 311-312.

Epstein, R. [A.], DeYoe, E. A., Press, D. Z., Rosen, A. C., \& KanWISHER, N. (2001). Neuropsychological evidence for a topographical learning mechanism in parahippocampal cortex. Cognitive Neuropsychology, 18, 481-508.
Epstein, R. A., Higgins, J. S., \& Thompson-Schill, S. L. (2005). Learning places from views: Variation in scene processing as a function of experience and navigational ability. Journal of Cognitive Neuroscience, 17, 73-83.

Fernald, A., \& MoriKawa, H. (1993). Common themes and cultural variations in Japanese and American mothers' speech to infants. Child Development, 64, 637-656.

Gardner, W. L., Gabriel, S., \& Lee, A. Y. (1999). "I" value freedom, but "we" value relationships: Self-construal priming mirrors cultural differences in judgment. Psychological Science, 10, 321-326.

Gernsbacher, M. A., \& KaschaK, M. P. (2003). Neuroimaging studies of language production and comprehension. Annual Review of Psychology, 54, 91-114.

Goh, J. O. S., Siong, S. C., Park, D. C., Gutchess, A. H., Hebrank, A., \& Chee, M. W. L. (2004). Cortical areas involved in object, background and object-background processing revealed with functional magnetic resonance adaptation. Journal of Neuroscience, 24, 1022310228.

GoNG, Y. (1983). Revision of Wechsler's Adult Intelligence Scale in China. Acta Psychologica Sinica, 15, 362-370.

Grossman, M., Koenig, P., DeVita, C., Glosser, G., Alsop, D., Detre, J., \& Gee, J. (2002). The neural basis for category-specific knowledge: An fMRI study. NeuroImage, 15, 936-948.

Hedden, T., Park, D. C., Nisbett, R., Ji, L., Jing, Q., \& Jiao, S. (2002). Cultural variation in verbal versus spatial neuropsychological function across the life span. Neuropsychology, 16, 65-73.

Holmes, A. P., \& Friston, K. J. (1998). Generalisability, random effects and population inference. NeuroImage, 7, S754.

Hong, Y., Morris, M. W., ChiU, C., \& Benet-Martinez, V. (2000). Multicultural minds: A dynamic constructivist approach to culture and cognition. American Psychologist, 55, 709-720.

IMAI, M., \& GENTNER, D. (1997). A cross-linguistic study of early word meaning: Universal ontology and linguistic influence. Cognition, 62, 169-200

IshiI, K., Reyes, A., \& Kitayama, S. (2003). Spontaneous attention to word content versus emotional tone: Differences among three cultures. Psychological Science, 14, 39-46.

Joseph, J. E., \& GATHERS, A. D. (2003). Effects of structural similarity on neural substrates for object recognition. Cognitive, Affective, \& Behavioral Neuroscience, 3, 1-16.

Kitayama, S., Duffy, S., Kawamura, T., \& Larsen, J. T. (2003). Perceiving an object and its context in different cultures: A cultural look at new look. Psychological Science, 14, 201-206.

Maguire, E. A., Gadian, D. G., Johnsrude, I. S., Good, C. D., AshBURner, J., Frackowiak, R. S., \& Frith, C. D. (2000). Navigationrelated structural change in the hippocampi of taxi drivers. Proceedings of the National Academy of Sciences, 97, 4398-4403.

Martin, A., Wiggs, C. L., Ungerleider, L. G., \& Haxby, J. V. (1996). Neural correlates of category-specific knowledge. Nature, 379, 649652.

Masuda, T., \& Nisbett, R. E. (2001). Attending holistically vs. analytically: Comparing the context sensitivity of Japanese and Americans. Journal of Personality \& Social Psychology, 81, 922-934.

Miyamoto, Y., Nisbett, R. E., \& Masuda, T. (2006). Culture and the physical environment: Holistic versus analytic perceptual affordances. Psychological Science, 17, 113-119.

NisBetT, R. E. (2003). The geography of thought: How Asians and Westerners think differently . . and why. New York: Free Press.

Nisbett, R. E., \& Masuda, T. (2003). Culture and point of view. Proceedings of the National Academy of Sciences, 100, 11163-11170.

Nisbett, R. E., Peng, K., Choi, I., \& Norenzayan, A. (2001). Culture and systems of thought: Holistic versus analytic cognition. Psychological Review, 108, 291-310.

PARK, D. C., \& Gutchess, A. H. (2002). Aging, cognition, and culture: A neuroscientific perspective. Neuroscience \& Biobehavioral Reviews, 26, 859-867.

Park, D. C., Hedden, T., Jing, Q., Jiao, S., Lautenschlager, G., \& NisBetT, R. E. (2005). Culture and the aging mind. Unpublished manuscript, University of Illinois, Urbana-Champaign.

Phan, K. L., Wager, T., Taylor, S. F., \& Liberzon, I. (2002). Func- 
tional neuroanatomy of emotion: A meta-analysis of emotion activation studies in PET and fMRI. NeuroImage, 16, 331-348.

PolK, T. A., \& FARAH, M. J. (1995). Late experience alters vision. Nature, 376, 648-649.

Polk, T. A., Stallcup, M., Aguirre, G. K., Alsop, D. C., D’EspoSito, M., Detre, J. A., \& FARAH, M. J. (2002). Neural specialization for letter recognition. Journal of Cognitive Neuroscience, 14, 145-159.

Postle, B. R., Awh, E., Jonides, J., Smith, E. E., \& D'Esposito, M. (2004). The where and how of attention-based rehearsal in spatial working memory. Cognitive Brain Research, 20, 194-205.

Puce, A., Allison, T., Asgari, M., Gore, J. C., \& McCarthy, G. (1996). Differential sensitivity of human visual cortex to faces, letterstrings, and textures: A functional magnetic resonance imaging study. Journal of Neuroscience, 16, 5205-5215.

Salthouse, T. A. (1996). General and specific speed mediation of adult age differences in memory. Journal of Gerontology: Psychological Sciences, 51, P30-P42.

Slotnick, S. D., Moo, L. R., Segal, J. B., \& Hart, J. (2003). Distinct prefrontal cortex activity associated with item memory and source memory for visual shapes. Cognitive Brain Research, 17, 75-82.

SNODGRAss, J. G., \& CoRWIN, J. (1988). Pragmatics of measuring recognition memory: Applications to dementia and amnesia. Journal of Experimental Psychology: General, 117, 34-50.

Sommer, T., Rose, M., Weiller, C., \& Büchel, C. (2005). Contributions of occipital, parietal, and parahippocampal cortex to encoding of object-location association. Neuropsychologia, 43, 732-743.

UNGERLEIDER, L. G. (1995). Functional brain imaging studies of cortical mechanisms for memory. Science, 270, 769-775.
WeCHSLER, D. (1981). Wechsler Adult Intelligence Scale-Revised. San Antonio, TX: The Psychological Corporation.

Whatmough, C., Chertkow, H., Murtha, S., \& Hanratty, K. (2002). Dissociable brain regions process object meaning and object structure during picture naming. Neuropsychologia, 40, 174-186.

Woods, R. P., Cherry, S. R., \& Mazziotta, J. C. (1992). Rapid automated algorithm for aligning and reslicing PET images. Journal of Computer Assisted Tomography, 16, 620-633.

\section{NOTE}

1. Although the lack of cultural differences may be surprising on the basis of previous behavioral studies (e.g., Masuda \& Nisbett, 2001), the extended encoding time of $4 \mathrm{sec}$ allows participants extensive time to encode aspects of the pictures. In a follow-up behavioral study of 24 American and 24 East Asian participants matched for age and years of education, performance was lowered with a 2-sec encoding window. Under these conditions, cultural differences emerged, with Americans performing better than Asians on recognition of pictures containing an object. There were significant cultural differences on object-only pictures $[t(46)=2.73, p<.01]$ and combined pictures $[t(46)=2.14, p<$ $.05]$ but only a marginal cultural difference for the background-only pictures $[t(46)=1.84, p>.07]$.

(Manuscript received July 25, 2005; revision accepted for publication December 6, 2005.) 